\title{
Evidence of double-loop hysteresis in disordered ferroelectric crystal
}

Cite as: J. Appl. Phys. 127, 184107 (2020); https://doi.org/10.1063/1.5143524

Submitted: 24 December 2019 . Accepted: 29 April 2020 . Published Online: 12 May 2020

Jacopo Parravicini (D), Lucia Fornasari, Eugenio DelRe, Franco Marabelli, Aharon J. Agranat, and Gianbattista Parravicini

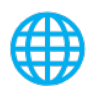

\section{Sign up for topic alerts} New articles delivered to your inbox 


\title{
Evidence of double-loop hysteresis in disordered ferroelectric crystal
}

\author{
Cite as: J. Appl. Phys. 127, 184107 (2020); doi: $10.1063 / 1.5143524$ \\ Submitted: 24 December 2019 . Accepted: 29 April 2020 . \\ Published Online: 12 May 2020
}

Jacopo Parravicini, ${ }^{1,2, a)}$ (D) Lucia Fornasari, ${ }^{3}$ Eugenio DelRe, ${ }^{4}$ Franco Marabelli, ${ }^{3}$ Aharon J. Agranat, ${ }^{5}$ and Cianbattista Parravicini ${ }^{3}$

\author{
AFFILIATIONS \\ 'Dipartimento di Scienza dei Materiali, Università di Milano-Bicocca, IT-20125 Milano, Italy \\ ${ }^{2}$ Erasmus Centre for Innovation, NL-3062PA Rotterdam, The Netherlands \\ ${ }^{3}$ Dipartimento di Fisica, Università di Pavia, IT-27100 Pavia, Italy \\ 4Dipartimento di Fisica, "Sapienza" Università di Roma, IT-00185 Roma, Italy \\ ${ }^{5}$ Applied Physics Department, Hebrew University of Jerusalem, IL-91904 Jerusalem, Israel
}

a) Author to whom correspondence should be addressed: jacopo.parravicini@unimib.it

\begin{abstract}
Double-loop electric-field vs polarization hysteresis is investigated in a depoled compositionally disordered lithium-enriched potassium tantalate niobate crystal. Comparing electro-optic response and dielectric spectroscopy indicates that the anomalous response occurs for those temperatures in which the sample also manifests a temperature hysteresis in the low-frequency dielectric function. An electric-field hysteresis at concurrent temperatures suggests an underlying role of reorienting mesoscopic polar regions that accompany the nonergodic phase.
\end{abstract}

Published under license by AIP Publishing. https://doi.org/10.1063/1.5143524

\section{INTRODUCTION}

Perovskite crystals are the object of a vast research effort because they operate as highly functional materials in a wide class of applications, ranging from dielectrics and capacitive elements, optical and photonic components, photovoltaic devices to piezoelectric sensors, while their physics is not altogether established. Among their useful properties, a widely exploited phenomenon is the electro-optic (EO) effect, which is often correlated with the typical ferroelectric nature of these compounds. ${ }^{1,2}$ In poled ferroelectric crystals, strong spontaneous polarization causes the EO response to be linear as a function of the applied field, while depoled or paraelectric crystals manifest a quadratic response; that is, the bias-induced change of the index of refraction is proportional to the square of the field. One basic example is a ferroelectric perovskite in a poled low-temperature tetragonal phase, where the effect is linear, so that, when heated into the high-temperature cubic phase, it manifests a quadratic effect. ${ }^{1}$ A different picture emerges when perovskite crystals host a given degree of disorder. Here, the EO response can be more complex and dependent on previous history, i.e., previous pressure, bias electric field, and temperature cycles. Specific thermal trajectories have been shown to lead to, for example, the enhancement of the electro-optic response, ${ }^{3,4}$ enhanced nonlinear response supporting scale-free optics and subwavelength beam propagation, ${ }^{5-7}$ marked order-disorder anisotropies, ${ }^{8,9}$ programable hysteretic effects, ${ }^{10-12,17}$ aging and rejuvenation, ${ }^{18,19}$ along with the presence of intrinsic nonlinearities and effective temperatures. ${ }^{20}$ One particularly striking and interesting signature of hysteretic phenomenology is the so-called double-loop isothermal paths as a function of the bias field. ${ }^{21-24}$

A key role in the interpretation of hysteretic effects is generally attributed to polar-nanoregions (PNRs) and the formation of a dipolar glass, a picture that is widely used in the modeling of disordered ferroelectrics. ${ }^{12,25,26}$ These arise as a consequence of ionic substitutional disorder in the crystalline lattice: a great number of defects strongly facilitate the formation of mesoscopic dipoles. Actually, the nature, concentration, and arrangement of these defects essentially determine the macroscopic physical quantities due to polarization behavior. In potassium-based perovskites, this was also found in the EO response, which was demonstrated to be strongly influenced by a small amount of $\mathrm{Li}$ content. ${ }^{13}$ Furthermore, in perovskites of the same family, defect content and features can be used to program the hysteretic behavior of polarization-field paths. ${ }^{14,16}$ The correlated PNRs are believed to become dominant when the system undergoes specific thermal trajectories in 
correspondence to the diffuse transition region, which is typical of relaxors. ${ }^{2,10,15,19,25-30}$ As in other glass-forming systems, the response of near-transition disordered ferroelectric samples is affected by the cooling or heating rate so that specific effects are observed only for a sufficiently rapid cooling or heating to the operating temperature. ${ }^{6,10,11,19,24,31,32}$ For example, optical scattering at the Curie point $T_{C}$ is diminished after rapid cooling, as is known to occur for glass-forming systems, where super-cooling suppresses the long-range order and macroscopic clusterization typical of conventional equilibrium phase-transitions. ${ }^{33,34}$ From a photonic perspective, this circumvents critical opalescence and allows optical transmission experiments to tap into the anomalously enhanced response that occurs during the paraelectric-to-ferroelectric transition. ${ }^{5,6,35}$

Experiments in disordered ferroelectrics have identified a range of temperatures spanning across $T_{C}$ where a non-ergodic evolution is found..$^{8-11,18,19,25,26,30,31}$ However, a comparative study of these features as a function of both temperature and electric-field bias has never been reported. In this work, we simultaneously investigate the temperature-dependent dielectric response and fielddependent electro-optic (EO) response. Specifically, operating at temperatures close to the ferroelectric transition, we electrooptically investigate double-loop hysteresis. We find that the double-loop response occurs only in the temperature region where non-ergodic thermal hysteresis is observed in the low-frequency dielectric susceptibility.

\section{EXPERIMENTAL PROCEDURES}

\section{A. Sample}

Our experiments are performed in potassium lithium tantalate niobate (KLTN), a compositionally disordered perovskite ferroelectric crystal. In our investigation, the KLTN sample is a single crystal of lithium-enriched potassium tantalate niobate $\left(\mathrm{K}_{1-y} \mathrm{Li}_{y} \mathrm{Ta}_{1-x} \mathrm{Nb}_{x} \mathrm{O}_{3}\right)$ with $x=0.36$ and $y=0.003$, which has been grown using the top-seeded-solution method. It is a $c$-direction-pulled zero-cut with sizes of $3.87(\mathrm{a}) \times 3.55(\mathrm{~b}) \times 1.35(\mathrm{c}) \mathrm{mm}^{8}$

\section{B. Dielectric measurements}

Dielectric spectroscopy is performed measuring the relative real dielectric permittivity $\varepsilon_{r}$ as a function of the temperature $T$ through a precision LCR meter (Agilent-4284A) by applying a probing $1 \mathrm{~V} / \mathrm{cm}$ field between plane-parallel electrodes deposited on the crystal facets ( $x$ direction). Temperature variation in the $255-295 \mathrm{~K}$ range (straddling the nominal phase transition $^{8}$ ) is achieved using a $T$-controlled closed two-stage helium cryostat and is monitored through a calibrated silicon diode sensor $(0.01 \mathrm{~K}$ in precision). Slow cooling and heating rates $(|\Delta T / \Delta t| \simeq 0.01 \mathrm{~K} / \mathrm{s})$ are employed in these measurements. ${ }^{10}$ The system records a measurement for each $0.25 \mathrm{~K}$ temperature variation. We recall that the obtained measurement gives the global response of the overall sample; i.e., it provides a dielectric response, which averages the contributions of local polarization anisotropies. Results can be discussed in terms of the Fröhlich entropy, an analysis that highlights order-disorder evolution. This exploits the relationship between $\varepsilon_{r}(T)$ and the entropy variation induced by the application of an electric field $\mathscr{E}$ (the fixed probing field in our case), which was provided by Fröhlich. ${ }^{41-43}$ The entropy variation $S_{\mathscr{E}}$ of a dielectric subjected to $\mathscr{E}$ is given, for the volume unit, by the relationship $S_{\mathscr{E}}=\left(\varepsilon_{0} / 2\right)\left(\partial \varepsilon_{r} / \partial T\right) \mathscr{E}^{2}$, where $\varepsilon_{0}$ is the vacuum permittivity, $\varepsilon_{r}$ is the real part of the dielectric function in the low-frequency limit, and $T$ the absolute temperature. It is useful to consider this last equation for the unitary field $s=S_{\mathscr{E}} / \mathscr{E}^{2}$, i.e.,

$$
s=\frac{\varepsilon_{0}}{2} \frac{\partial \varepsilon_{r}}{\partial T},
$$

where $s=s(T)$ is the Fröhlich entropy for the unitary volume and field. $^{8,43,44}$ Equation (1) shows that the application of $\mathscr{E}$ increases the entropy if $s(T)>0$ and decreases the entropy if $s(T)<0$. Actually, an external field creates order in a dipolar liquid-like system because it will orientate some of the random dipoles and the entropy variation is negative; on the other hand in a dipolar ordered system, the field increases disorder with a positive value for $s(T){ }^{8,9}$

\section{Electro-optic measurements}

The electro-optic (EO) response is investigated using a crosspolarizer transmission experiment: the birefringent sample is sandwiched between two crossed polarizers, the first transmitting light polarized at $45^{\circ}$ to the $x$ axis while the second transmits light at $-45^{\circ}$, and laser light transmission is measured as a function of the bias electric field $E$, along the $x$ direction, and temperature $T .{ }^{20}$ The transmitted light intensity $I$ then obeys the relationship,

$$
I=I_{0}+I_{1} \sin ^{2}(\Delta \phi / 2),
$$

where $I_{0}$ is the intensity due to the residual birefringence, $I_{1}$ is the minimum of the modulated intensity, and $\Delta \phi$ is the field-induced phase-shift between the $x$ and $y$ optical field components. The shift is then connected to the field-induced sample birefringence $\Delta \phi=L(2 \pi / \lambda) \Delta n$, where $\lambda$ is the wavelength of light, $L$ is the length of the crystal along $z$, and $\Delta n(E)=n_{x x}-n_{y y}$ is the field-induced difference between the index of refraction for light polarized along the $x$ and $y$ direction. In general, the change in the index of refraction for an input field polarized along the $i$ axis and exit polarization along the $j$ axis is

$$
\Delta n_{i j}=-\frac{1}{2} n_{0}^{3} g_{i j k l} P_{k} P_{l},
$$

where $g_{i j k l}$ is the quadratic electro-optic 4-tensor and $P_{k}$ is the $k$ component of the material polarization $(i, j, k, l$ label the corresponding $x, y, z$ directions of the involved fields and permittivity tensors), which then depends on the specific constitutive relation $\mathbf{P}=\mathbf{P}(\mathbf{E})$. Hence, investigating $\Delta \phi$, or equivalently $\Delta n=\Delta \phi(\lambda / 2 \pi L)$, as a function of $\mathbf{E}$, we are able to optically measure the $P$ vs $E$ relationship at the given temperature $T$.

For example, in the cubic (paraelectric) phase, in conditions in which $P_{i}=\varepsilon_{0} \varepsilon_{r} E_{i}$, with $\varepsilon_{r}$ being a scalar (in our experiments, $\varepsilon_{r}-1 \simeq \varepsilon_{r}$; i.e., the relative dielectric constant is $\varepsilon_{r} \gg 1$ ), we 
expect that

$$
\Delta n_{\mathrm{cub}}(E)=-\frac{1}{2} n_{0}^{3} g_{\mathrm{eff}} \varepsilon_{0}^{2} \varepsilon_{r}^{2} E^{2}
$$

Here, $n_{0}$ is the unperturbed index of refraction $\left(n_{0} \simeq 2.31\right.$ in our experiment), and $g_{\text {eff }} \equiv\left(g_{11}-g_{12}\right)=0.14 \mathrm{C}^{-2} \mathrm{~m}^{4}$ is the effective quadratic EO coefficient for the nominal $\mathrm{m} 3 \mathrm{~m}$ symmetry $\left(g_{11}=g_{x x x x}, g_{12}=g_{x x y y}\right)$. ${ }^{1,20,49}$

In turn, when the sample is below the transition temperature, in the nominally tetragonal phase, and poled along a given principal axis (say, the $x$ axis), the presence of a strong spontaneous polarization causes the leading EO dephasing to become linear and described by

$$
\Delta n_{\mathrm{tetr}}(E)=-\frac{1}{2} n_{0}^{3} r_{\mathrm{eff}} E,
$$

where $r_{\text {eff }}$ is the linear effective EO coefficient, suitably contracted and calculated with respect to the symmetry of the correlated tensor. ${ }^{1,49,50}$ Hence, when a standard crystalline transition occurs from the cubic to tetragonal phase, the $\mathrm{EO}$ response usually passes from a $\Delta n \propto E^{2}$ to a $\Delta n \propto E$.

The employed optical setup is shown in Fig. 1. A Gaussian beam from a He-Ne laser $(\lambda=632.8 \mathrm{~nm})$ is expanded to an approximate plane wave of $10 \mathrm{~mm}$ diameter, propagating along $z$, by a suitable beam expander. The linearly polarized light at $45^{\circ}$ with respect to the $x$ axis is transmitted by the first polarizer and passes through the sample. The $\lambda / 4$ waveplate allows us to suppress the $I_{0}$ intensity of the exiting beam [Eq. (2)] due to the residual birefringence. Then, the beam passes through the second polarizer, which is orthogonal to the first one. The zero-cut sample is aligned with its principal $\mathrm{m} 3 \mathrm{~m}$ axes along $x, y$, and $z$. Conductive electrodes are painted on the $x$-lateral facets, allowing the application of a static electric field $E$ along $x$. The temperature $T$ is varied using a controlled He-cryostat, whose cold finger is located immediately below the sample. The actual temperature is measured by a calibrated diode embedded in the sample holder, immediately below the sample. Transmitted light is collected by a lens, and the optical power is detected using a power meter (Thorlabs model PM100D with a Thorlabs S120C silicon power sensor). For the average plane-wave intensity impinging on the sample of $\simeq 0.5 \mathrm{~mW} / \mathrm{cm}^{2}$, no photorefractive effects for the duration of our experiments are detected. The electric field $E$ is delivered by a Stanford Research Model PS350 high-voltage power supply: it is programed to provide a voltage $\Delta V$ from 0 to $900 \mathrm{~V}$, with either a positive or negative sign. We highlight

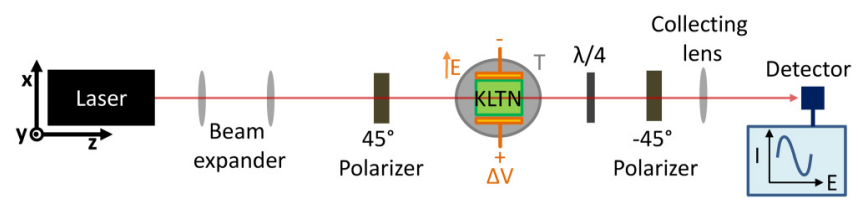

FIG. 1. Crossed-polarizer setup. that, similarly to dielectric experiments, this measurement of the EO response averages the different contributions along the whole sample.

\section{DIELECTRIC RESPONSE}

At high temperatures, the studied KLTN crystal has a cubic symmetry with a paraelectric behavior. ${ }^{8}$ When the temperature $T$ is lowered, at a given so-called Burns temperature $T_{B},{ }^{36}$ the disordered distribution of inherent off-center ions, which occupy energetically equivalent lattice sites, leads to the formation of mesoscopic polar regions (PNRs) that below a temperature $T^{*}<T_{B}$ are permanent and distributed in the highly polarizable lattice. They are uncorrelated and free to reorient, while both their number and size increase on further lowering temperature. ${ }^{25,26,29,30,37,38}$

At still lower temperature, relaxor ferroelectrics may undergo a sharp or a diffuse (as in our sample) relaxor-to-ferroelectric phase transition (according to the Bokov classification) ${ }^{26}$ with the transformation of the long-range crystalline symmetry from cubic to tetragonal. Specifically, at a critical temperature, here indicated with $T_{m}$, the PNRs correlate through a percolative process so giving rise to a polar glassy-like state ${ }^{38-40}$ that evolves in temperature to a ferroelectric state.

In Fig. 2(a), the relative real part of the dielectric permittivity (frequency $10 \mathrm{kHz}) \varepsilon_{r}=\varepsilon_{r}(T)$ is reported both for cooling and heating strokes. The wide peaks signal the transition from the cubic (high $T$ ) to tetragonal (low $T$ ) symmetry. ${ }^{8,26,29}$ Evident thermal hysteresis in $\varepsilon_{r}(T)$ is found from $T \simeq 275 \mathrm{~K}$ to $T=289 \mathrm{~K}$, which we call the cross-over region, while the peak temperatures are $T_{m}=285 \mathrm{~K}$ and $T_{m}=287 \mathrm{~K}$ on cooling and heating, respectively. This hysteretic behavior is a signature of an out-of-thermodynamic-equilibrium non-ergodic phase that characterizes the cross-over region. It is worth considering that a nonergodic phase is thermal and field history dependent. In the same figure, the shaded region signals the non-ergodic phase several degrees above and below the critical temperatures $T_{m}$, a well-known complex dielectric response ${ }^{34,45,46}$ that is analogous to that observed in relaxors of the same family, ${ }^{25-28}$ where we expect glassy-state correlated effects. ${ }^{19}$

In Fig. 2(b), the Fröhlich entropy as a function of temperature $s(T)$ is reported. In the high-temperature region $(T \gtrsim 289 \mathrm{~K})$, the negative sign of $s(T)$ marks the dipolar disorder in the sample due to uncorrelated PNRs that increases with lowering the temperature as expected. ${ }^{8,9,37,41}$ An inversion of the negative trend of $s(T)$ is displayed at $289 \mathrm{~K}$ and $287 \mathrm{~K}$ for heating and cooling strokes, respectively. By lowering the temperature, an abrupt change of the $s(T)$ sign from negative to large positive values occurs, for both the strokes, in correspondence to the relative maxima of the permittivity. These findings are the signature of a sudden onset of a different state of order in the system (disorder/order transition), as a result of a new arrangement of PNRs that cease to be uncorrelated and give rise to a more structured (ordered) dipolar configuration. The region where $s(T)$ assumes positive values shows the occurrence of strong instability, where order and disorder are competing and the physical system is reconfiguring. ${ }^{8,47,48}$ Below this transition, the evolution in the temperature of $s(T)$ shows that the ferroelectric phase does not achieve a stable configuration up to $\simeq 275 \mathrm{~K}$, where 

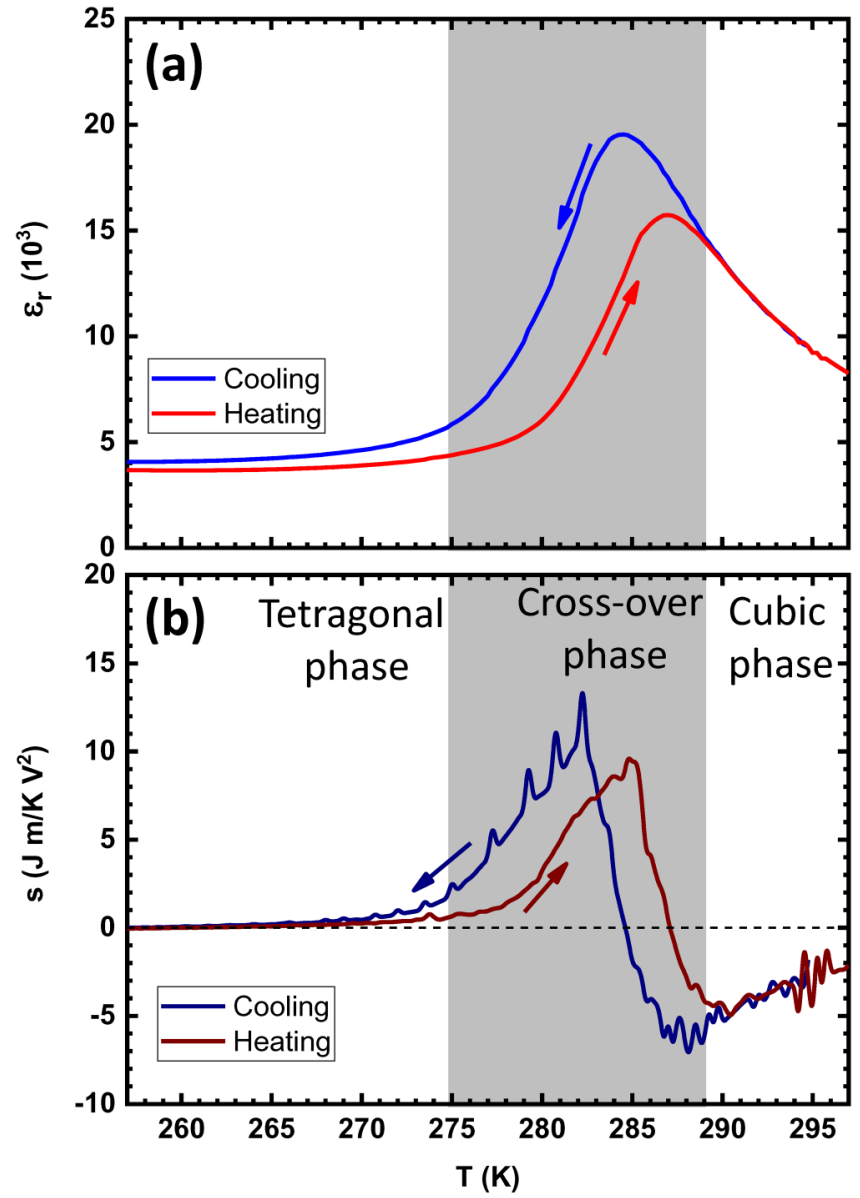

FIG. 2. (a) KLTN relative permittivity $\varepsilon_{r}$ for cooling (blue curve) and heating (red curve) as a function of temperature. (b) Calculated Fröhlich entropy $s=s(T)$ with the indicated transition (or cross-over) region. The measurement rate is one recorded point for each $0.25 \mathrm{~K}$. the permittivity curves become very close. This phase instability is highlighted by the oscillations in the cooling stroke, confirming the non-ergodic state of the cross-over region. It is worth considering that, unlike the diffused ferroelectric transition, the sharp one immediately below the transition critical temperature attains a stable ferroelectric phase where non-ergodic effects are absent. ${ }^{8,42}$

\section{ELECTRO-OPTIC RESPONSE}

In our EO experiments, we consider the temperature region from 260 to $305 \mathrm{~K}$ with approximatively one measurement for each degree. The measurement procedure is as follows: the sample is subject to a specific four-sweep bias cycle during which the transmitted light intensity (Fig. 1) is recorded at each $10 \mathrm{~V}$ interval. Each sweep requires roughly $450 \mathrm{~s}$ to be completed. In the first one (which we call the 1st forward), while the sample is maintained at a given temperature, $\Delta V$ is made to increase from 0 to $+900 \mathrm{~V}$; then, $\Delta V$ is decreased from +900 to $0 \mathrm{~V}$ (the second sweep, the $1 s t$ backward). Thereafter, the voltage is varied from 0 to $-900 \mathrm{~V}$ (the third sweep, the 2nd forward); and finally, $\Delta V$ returns from -900 to $0 \mathrm{~V}$ (the fourth sweep, the 2 nd backward). After this, the temperature of the sample is varied and the above-described procedure is repeated. The entire temperature range is spanned three times and the acquired data are averaged. Then, from the transmitted light intensity signals, the induced dephasing $\Delta \phi=\Delta \phi(E)$ is calculated, at each temperature $T$, with the same procedure as in Ref. 20. We note that in the measured interferometric intensity pattern, in the nominally tetragonal crystalline phase, positive and negative field modulations are in counterphase, and they are in phase in the nominally cubic crystalline region [Eq. (2)], as expected.

In order to investigate the presence of specific time dynamics in the EO response within our considered temporal range, we have also tested bias sweeps with different time durations: namely, we have considered intervals from approximatively $300 \mathrm{~s}$ to $1000 \mathrm{~s}$ per sweep. Moreover, we have checked the presence of changes in time during the application of a constant field by maintaining the sample under the maximum field strength for roughly $200 \mathrm{~s}$. In
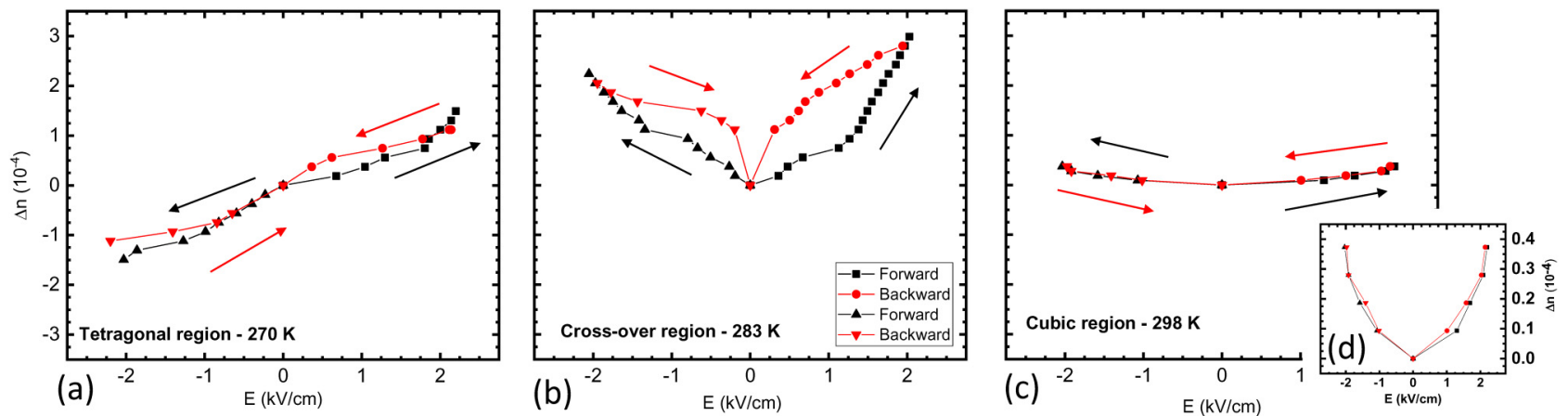

FIG. 3. Typical electro-optic measurements in the three temperature regions: (a) tetragonal phase, (b) cross-over phase, and (c) cubic phase [with its correlated zoom in (d)]. The relative errors in $E$ and $\Delta n$ are of $3 \%$ in (a), $4 \%-5 \%$ in (b), and $5 \%-6 \%$ in (c) and (d). 


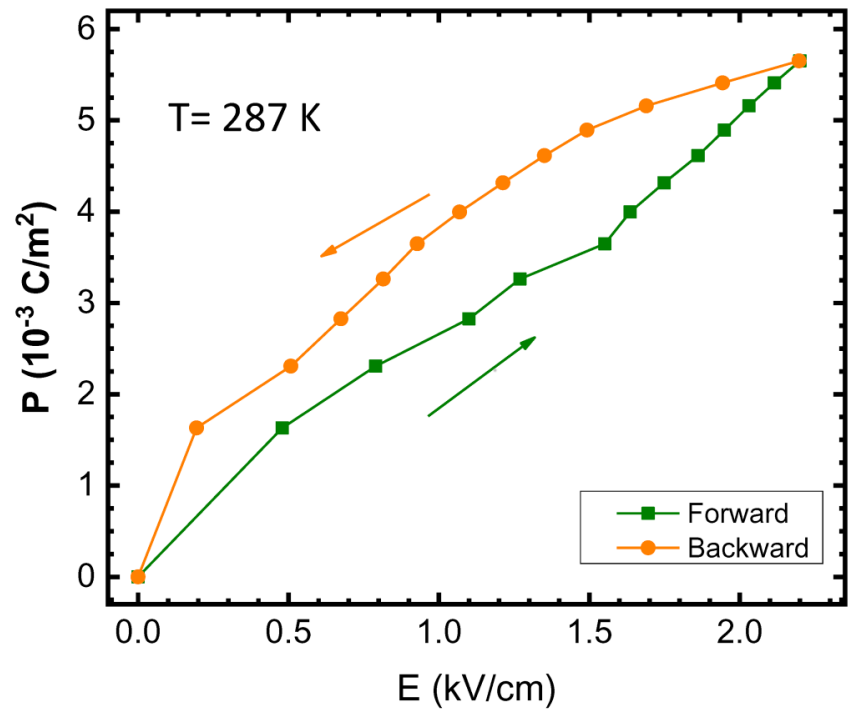

FIG. 4. The $P$ vs $E$ relationship in the cross-over temperature region from the measured values of $\Delta n(E)$ [from Eq. (6)] indicates the induced field-hysteretic electro-optic loop

both cases, no significant evolutions correlated with the time have been evidenced in our considered experimental time scales.

In Fig. 3, we report typical results where the field application cycle composed of four sweeps is evidenced (1st forward, 1st backward, etc.). Data for three temperatures are reported: the first temperature [Fig. 3(a)] within the low-temperature-region of the plot in 2(b) (tetragonal, ordered phase), the second one [Fig. 3(b)] belonging to the cross-over region, and the third one [Fig. 3(c)] in the cubic (disordered) phase.

We first discuss the conditions of the tetragonal and the cubic phases. In Fig. 3(a), a measurement of the EO effect of our KLTN sample at $270 \mathrm{~K}$ is plotted. Here, the dependence of $\Delta n$ on the applied electric field is linear as expected. Furthermore, the cycle of field application does not display significant hysteretic features. At the other extreme, Fig. 3(c), which is performed at $T=297 \mathrm{~K}$, shows a quadratic dependence, which, within the experimental error, can be easily described by Eq. (4). Again, no hysteretic effects can be recognized.

Investigating the intermediate cross-over temperature region, in-between the previous ones, we find a clear cross-polarizer transmission pattern in the form of relationship (2), indicating that critical scattering plays a negligible role for EO measurements. Remarkably, the detected response $\Delta n$ vs $E$ is profoundly different from both linear and quadratic electro-optic effects. The experiment at the sample temperature of $T=283 \mathrm{~K}$ is reported in Fig. 3(b). We also point out that the curves in the plot of Fig. 3(b) draw a typical butterfly-like pattern similar to that found in other ferroelectric crystals. ${ }^{12,17,21}$ When the electric-field cycle is repeated, by following the same four sweeps, approximately, the same response is found. This indicates that the obtained evolution is reproducible and that the application of the electric field does not induce a permanent transition in the system, either toward the tetragonal or cubic phase. The double-loop trend is maintained in the whole cross-over temperature range pointed out by the dielectric response. In Fig. 4, the $P$ vs $E$ modulation, calculated from $\Delta n$ EO measurements, is given by ${ }^{20}$

$$
P=\sqrt{\left(-2 \Delta n(E) / n_{0}^{3}\right)\left(g_{11}-g_{12}\right)} .
$$

In Fig. 4, the trend of $P$ is reported, for positive values of the applied field, at $T=287 \mathrm{~K}$, a slightly higher temperature with respect to what is depicted in Fig. 3(b). We note that $P=P(E)$ shows a very different field dependence in forward with respect to the backward sweep. This is further evidenced in Fig. 4, where the behavior of the polarization $P$ provided from EO measurements at a second temperature in the cross-over region is reported, which confirms the observed history-dependent behavior.

\section{CONCLUSIONS}

In summary, we have demonstrated how in a disordered ferroelectric, the cross-over temperature region between nominally tetragonal and cubic phases leads to peculiar dynamics in the EO response. For temperatures in the transition region, the hysteresis in the temperature is accompanied by a hysteresis in polarization vs the electric field. Specifically, the double-loop pattern is the signature of the passage from the quadratic to the linear EO effect. This is manifested by a characteristic double-loop response, which is analogous to what is already found in other ferroelectric perovskites.

\section{ACKNOWLEDGMENTS}

E.D. acknowledges support from the ATTRACT SALT grant and from the Sapienza Ateneo 2018 project. J.P. and G.P. thank M. Moscardini for technical assistance.

\section{REFERENCES}

${ }^{1}$ R. Boyd, Nonlinear Optics, 3rd ed. (Academic Press, Cambridge, MA, 2008).

${ }^{2}$ F. Jona and G. Shirane, Ferroelectric Crystals (Dover, New York, 1993).

${ }^{3}$ Y.-C. Chang, C. Wang, S. Yin, R. C. Hoffman, and A. G. Mott, "Kovacs effect enhanced broadband large field of view electro-optic modulators in nanodisordered KTN crystals," Opt. Express 21, 17760-17768 (2013).

${ }^{4}$ Y.-C. Chang, C. Wang, S. Yin, R. C. Hoffman, and A. G. Mott, "Giant electrooptic effect in nanodisordered KTN crystals," Opt. Lett. 38, 4574-4577 (2013).

${ }^{5}$ E. DelRe, E. Spinozzi, A. J. Agranat, and C. Conti, "Scale-free optics and diffractionless waves in nanodisordered ferroelectrics," Nat. Photonics 5, 39 (2011)

${ }^{6}$ E. DelRe, F. Di Mei, J. Parravicini, G. B. Parravicini, A. J. Agranat, and C. Conti, "Subwavelength anti-diffracting beams propagating for more than 1,000 Rayleigh lengths," Nat. Photonics 9, 228 (2015).

${ }^{7}$ J. Parravicini, P. Minzioni, V. Degiorgio, and E. DelRe, "Observation of nonlinear airy-like beam evolution in lithium-niobate," Opt. Lett. 34, 3908 (2009).

${ }^{8}$ J. Parravicini, E. DelRe, A. J. Agranat, and G. B. Parravicini, "Macroscopic response and directional disorder dynamics in chemically substituted ferroelectrics," Phys. Rev. B 93, 094203 (2016).

${ }^{9}$ J. Parravicini, E. DelRe, A. J. Agranat, and G. B. Parravicini, "Liquid-solid directional composites and anisotropic dipolar phases of polar nanoregions in disordered perovskite," Nanoscale 9, 9572-9580 (2017). 
${ }^{10}$ J. Parravicini, C. Conti, A. J. Agranat, and E. DelRe, "Programming scale-free optics in disordered ferroelectrics," Opt. Lett. 37, 2355-2357 (2012).

${ }^{11}$ J. Parravicini, A. J. Agranat, C. Conti, and E. DelRe, "Equalizing disordered ferroelectrics for diffraction cancellation," Appl. Phys. Lett. 101, 111104 (2012).

${ }^{12}$ H. Tian, B. Yao, P. Tan, Z. Zhou, G. Shi, D. Gong, and R. Zhang, "Double-loop hysteresis in tetragonal $\mathrm{KTa}_{0} .58 \mathrm{Nb}_{0} .42 \mathrm{O}_{3}$ correlated to recoverable reorientations of the asymmetric polar domains," Appl. Phys. Lett. 106, 102903 (2015).

${ }^{13}$ A. Gumennik, Y. Kurzweil-Segev, and A. J. Agranat, "Electrooptical effects in glass forming liquids of dipolar nano-clusters embedded in a paraelectric environment," Opt. Mat. Express 1, 332-343 (2011).

${ }^{14}$ X. Cao, H. Tian, C. Hu, Y. Wang, X. Li, L. Li, X. Sun, and Z. Zhou, "Discovery and evolution of double $P-E$ loops in a tetragonal Fe-doped $\mathrm{KTa}_{0} \cdot 57 \mathrm{Nb}_{0} \cdot 43 \mathrm{O}_{3}$ single crystal," J. Am. Ceram. Soc. 101, 3755-3760 (2018).

${ }^{15}$ X. Cao, H. Tian, C. Hu, F. Huang, Y. Wang, X. Sun, and Z. Zhou, "Defect dipole evolution and its impact on the ferroelectric properties of Fe-doped KTN single crystals," J. Am. Ceram. Soc. 102, 3117-3122 (2019).

${ }^{16} \mathrm{H}$. Tian, F. Huang, X. Meng, Y. Wang, Z. Xian, C. Hu, and Z. Zhou, "Improvement of anti-fatigued strain in KTN-based single crystals with regular periodic hierarchical domain structure," Appl. Phys. Lett. 115, 172901 (2019).

${ }^{17}$ K. S. Lam, Y. Zhou, Y. W. Wong, and F. G. Shin, "Electrostriction of lead zirconate titanate/polyurethane composites,” J. Appl. Phys. 97, 104112 (2005).

${ }^{18}$ J. Parravicini, D. Pierangeli, F. Di Mei, C. Conti, A. J. Agranat, and E. DelRe, "Aging solitons in photorefractive dipolar glasses," Opt. Express 21, 30573 (2013).

${ }^{19}$ J. Parravicini, A. J. Agranat, C. Conti, and E. DelRe, "Rejuvenation in scale-free optics and enhanced diffraction cancellation life-time," Opt. Express 20, 27382 (2012).

${ }^{20}$ D. Pierangeli, F. Di Mei, J. Parravicini, G. B. Parravicini, A. J. Agranat, and E. DelRe, "Observation of an intrinsic nonlinearity in the electro-optic response of freezing relaxors ferroelectrics," Opt. Mater. Express 4, 1487 (2014).

${ }^{21}$ J. Î́ñiguez, P. Zubko, I. Luk'yanchuk, and A. Cano, "Ferroelectric negative capacitance," Nat. Rev. Mater. 4, 243-256 (2019).

${ }^{22}$ S. Gevorgian, A. Eriksson, A. Deleniv, and D. Pandey, "Double loop hysteresis in direct current dependent dielectric permittivity of $\mathrm{SrTiO}_{3}$," J. Appl. Phys. $\mathbf{9 2}$ 6165 (2002).

${ }^{23}$ D. Lin, K. W. Kwok, and H. L. Chan, "Double hysteresis loop in Cu-doped $\mathrm{K}_{0} .5 \mathrm{Na}_{0} .5 \mathrm{NbO}_{3}$ lead-free piezoelectric ceramics," Appl. Phys. Lett. 90, 232903 (2007).

${ }^{24}$ W. J. Merz, "Double hysteresis loop of $\mathrm{BaTiO}_{3}$ at the Curie point," Phys. Rev. 91, 513-517 (1953).

${ }^{25}$ P. Ben Ishai, C. E. M. de Oliveira, Y. Ryabov, Y. Feldman, and A. J. Agranat, "Glass-forming liquid kinetics manifested in a KTN:Cu crystal," Phys. Rev. B 70, 132104 (2004).

${ }^{26}$ A. A. Bokov and Z.-G. Ye, "Recent progress in relaxor ferroelectrics with perovskite structure”, J. Mater. Sci. 41, 31 (2006).

${ }^{27}$ B. E. Vugmeister and M. D. Glinchuk, "Dipole glass and ferroelectricity in random-site electric dipole systems," Rev. Mod. Phys. 62, 993 (1990).

${ }^{28}$ M. D. Glinchuk and V. A. Stephanovich, "The peculiarities of dielectric susceptibility dynamics in mixed ferro-glass phase of disordered ferroelectrics," Ferroelectr. Lett. 22, 113-119 (1997).

${ }^{29} \mathrm{G}$. A. Samara, "The relaxational properties of compositionally disordered $\mathrm{ABO}_{3}$ perovskites", J. Phys. Condens. Matter 15, R367 (2003).
${ }^{30}$ A. A. Bokov and Z.-G. Ye, "Dielectric relaxation in relaxor ferroelectrics," J. Adv. Dielectr. 2, 1241010 (2012).

${ }^{31}$ G. L. Yuan, Y. Yang, and S. W. Or, "Aging-induced double ferroelectric hysteresis loops in $\mathrm{BiFeO}_{3}$ multiferroic ceramic,” Appl. Phys. Lett. 91, 122907 (2007)

${ }^{32}$ M. D. Glinchuk and V. A. Stephanovich, "Dynamic properties of relaxor ferroelectrics," J. Appl. Phys. 85, 1722 (1999).

${ }^{33}$ E. DelRe, M. Tamburrini, M. Segev, R. Della Pergola, and A. J. Agranat, "Spontaneous self-trapping of optical beams in metastable paraelectric crystals," Phys. Rev. Lett. 83, 1954 (1999).

${ }^{34} \mathrm{~W}$. Kleemann and R. Lindner, "Dynamic behavior of polar nanodomains in $\mathrm{PbMg}_{1} / 3 \mathrm{Nb}_{2} / 3 \mathrm{O}_{3}$," Ferroelectrics 199, 1 (1997).

${ }^{35}$ F. Di Mei, L. Falsi, M. Flammini, D. Pierangeli, P. Di Porto, A. J. Agranat, and E. DelRe, "Giant broadband refraction in the visible in a ferroelectric perovskite," Nat. Photonics 12, 734-738 (2018).

${ }^{36} \mathrm{G}$. Burns and F. H. Dacol, "Crystalline ferroelectrics with glassy polarization behavior," Phys. Rev. B 28, 2527 (1983).

${ }^{37}$ V. V. Shvartsman and D. C. Lupascu, "Lead-free relaxor ferroelectrics," Am. Ceram. Soc. 95, 1-26 (2012)

${ }^{38} \mathrm{R}$. Pirc and Z. Kutnjak, "Electric-field dependent freezing in relaxor ferroelectrics," Phys. Rev. B 89, 184110 (2014)

${ }^{39}$ L. Cai, J. Toulouse, L. Harriger, R. G. Downing, and L. A. Boatner, "Origin of the crossover between a freezing and a structural transition at low concentration in the relaxor ferroelectric $\mathrm{K}_{1-x} \mathrm{Li}_{x} \mathrm{TaO}_{3}$," Phys. Rev. B 91, 134106 (2015).

${ }^{40}$ B. Rožič, M. Kosec, H. Uršič, J. Holc, B. Malič, Q. M. Zhang, R. Blinc, R. Pirc, and Z. Kutnjak, "Influence of the critical point on the electrocaloric response of relaxor ferroelectrics," J. Appl. Phys. 110, 064118 (2011).

${ }^{41} \mathrm{H}$. Fröhlich, Theory of Dielectrics (Clarendon Press, Oxford, 1958), Chap. 1.

${ }^{42} \mathrm{G}$. P. Johari, "Effects of electric field on the entropy, viscosity, relaxation time, and glass-formation," J. Chem. Phys. 138, 154503 (2013).

${ }^{43}$ J. Parravicini, "Thermodynamic potentials in anisotropic and nonlinear dielectrics," Physica B 541, 54-60 (2018).

${ }^{44}$ P. Tan, H. Tian, F. Huang, X. Meng, Y. Wang, C. Hu, X. Cao, L. Li, and Z. Zhou, "Strain-gradient controlled disorder dynamics in chemically substituted ferroelectrics," Phys. Rev. Appl. 11, 024037 (2019).

${ }^{45} \mathrm{~L}$. Leuzzi and T. M. Nieuwenhuizen, Thermodynamics of the Glassy State (Taylor \& Francis, 2008).

${ }^{46}$ S. Mossa and F. Sciortino, "Crossover (or Kovacs) effect in an aging molecular liquid,” Phys. Rev. Lett. 92, 045504-1 (2004).

${ }^{47}$ G. B. Parravicini, A. Stella, P. Tognini, P. G. Merli, A. Migliori, P. Cheyssac, and R. Kofman, "Insight into the premelting and melting processes of metal nanoparticles through capacitance measurements," Appl. Phys. Lett. 82, 1461 (2003).

${ }^{48}$ A. Sassella, D. Braga, M. Campione, T. Ciabattoni, M. Moret, J. Parravicini, and G. B. Parravicini, "Probing phase transitions and stability of organic semiconductor single crystals by dielectric investigation," J. Appl. Phys. 109, 013529 (2011).

${ }^{49}$ V. G. Dmitriev, G. G. Gurzadyan, and D. N. Nikogosyan, Handbook of Nonlinear Optical Crystals, 3rd ed. (Springer, Berlin, 1999).

${ }^{50}$ J. Parravicini, J. Safioui, M. Chauvet, P. Minzioni, and V. Degiorgio, "All-optical technique to measure the pyroelectric coefficient in electro-optic crystals,” J. Appl. Phys. 109, 033106 (2011). 